\title{
Equation of State for QCD at finite temperature and density. Resummation versus lattice data
}

\author{
Jens O. Andersen ${ }^{1 *}$, Najmul Haque ${ }^{\dagger}$, Munshi G. Mustafa ${ }^{\dagger}$, Michael Strickland ${ }^{* *}$ and \\ $\mathrm{Nan} \mathrm{Su}^{\ddagger}$
}

${ }^{*}$ Department of Physics, Norwegian University of Science and Technology, N-7491 Trondheim, Norway

${ }^{\dagger}$ Theory Division, Saha Institute of Nuclear Physics, 1/AF Bidhannagar, Kolkata-700064, India

${ }^{* *}$ Department of Physics, Kent State University, Kent, Ohio 44242, United States

${ }^{\ddagger}$ Fakultät für Physik, Universität Bielefeld, 33615 Bielefeld, Germany

\begin{abstract}
The perturbative series for finite-temperature field theories has very poor convergence properties and one needs a way to reorganize it. In this talk, I review two ways of reorganizing the perturbative series for field theories at finite temperature and chemical potential, namely hard-thermal-loop perturbation theory (HTLpt) and dimensional reduction (DR). I will present results for the pressure, trace anomaly, speed of sound and the quark susceptibilities from a 3-loop HTLpt calculation and for the quark susceptibilities using DR at four loops. A careful comparison with available lattice data shows good agreement for a number of physical quantities.
\end{abstract}

Keywords: QCD equation of state, Quark-gluon plasma, Hard-thermal-loop perturbation theory, Lattice QCD calculations. PACS: $64.30 . \mathrm{Ef}, 12.38 . \mathrm{Mh}, 12.38 . \mathrm{Gc}$.

\section{INTRODUCTION}

In this talk I would like to discuss a problem that has been around for a couple of decades namely the poor convergence of the perturbative series of the thermodynamic functions of hot and dense QCD. The weak-coupling expansion of the QCD pressure has a very long story going back to the late 1970s $[1,2,3,4,5,6,7,8]$ and the interest has in part been spurred by application to quark-gluon plasma phenomenology in heavy-ion collisions. The calculational frontier has been pushed to order $g^{6} \ln (g)$ at zero chemical potential [9] and finite chemical potential [10,11, 12].

In Fig. 1, we show the weak-coupling expansion of the QCD pressure with $N_{f}=3$ normalized to that of an ideal gas of quarks and gluons through order $g^{2}, g^{3}, g^{4}$, and $g^{5}$. The curves are obtained by using the strong coupling constant $g(\Lambda)$ evaluated at the renormalization scale $\Lambda=2 \pi T$. The bands are obtained by varying the renormalization scale by a factor of two around this central value. As successive terms in the weak-coupling expansion are added, the predictions for the pressure fluctuate wildly and the sensitivity to the renormalization scale $\Lambda$ grows. Due to asymptotic freedom, the weak-coupling expansion does converge for sufficiently high temperatures. However, this is only the case for temperatures many orders of magnitude larger than the critical temperature $T_{c}$ for deconfinement. For example the order $g^{3}$ term is smaller than the order- $g^{2}$ only if $T$ is larger than approximately $10^{5} T_{c}$. We note in passing that the poor convergence is not specific to QCD, it is a generic feature of hot quantum field theories. This together with the large $g^{3}$ corrections suggests that the problem is in the soft sector of the theory, i.e. momenta on the order of $g T$, and is not related to the breakdown of perturbation theory due to infrared divergences at four loops (Linde's problem) [13]. There are several ways of reorganizing the perturbative series [14, 15], here I will focus on hard-thermal-loop perturbation theory (HTLpt) and dimensional reduction (DR). The results presented in this talk are published in the papers $[16,17,18,19]$.

\footnotetext{
${ }^{1}$ Speaker.
} 


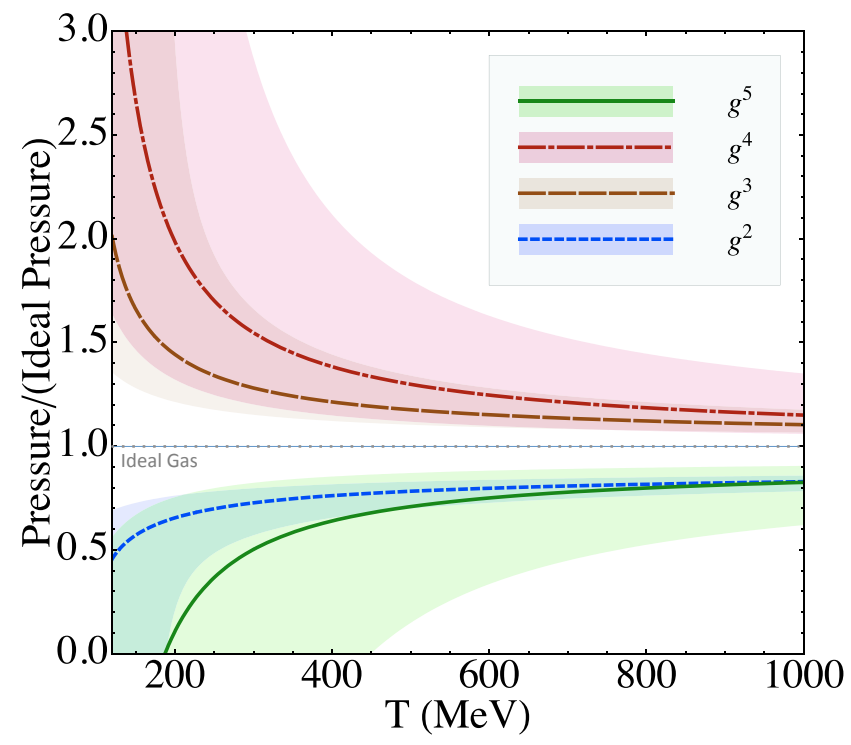

FIGURE 1. Weak-coupling expansion of the normalized pressure in three-flavor massless QCD as a function of the temperature $T$. See main text for details.

\section{REORGANIZATION OF THE PERTURBATIVE SERIES}

\section{Screened perturbation theory}

Before we discuss hard-thermal-loop perturbation theory in some detail, it is useful to take a step back and discuss resummation in a self-interacting scalar field theory. The starting point is the Euclidean Lagrangian for massless $\phi^{4}$ theory

$$
\mathscr{L}=\frac{1}{2}\left(\partial_{\mu} \phi\right)^{2}+\frac{g^{2}}{24} \phi^{4}
$$

We are interested in calculating perturbative corrections to various quantities, for example the two-point function. Fig. 2 shows diagrammatically the inverse propagator to one-loop order.

Calculating the one-loop diagram, the inverse propagator can be written as

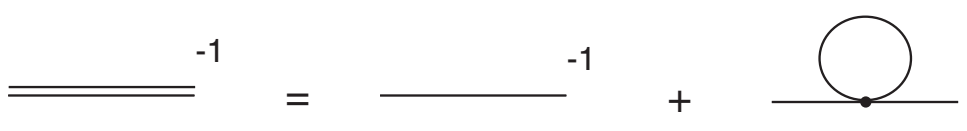

FIGURE 2. Inverse propagator to one-loop order.

$$
\Delta^{-1}=P^{2}+\frac{g^{2}}{24} T^{2},
$$

where $P=\left(P_{0}=\omega_{n}, \mathbf{p}\right)$. For hard momenta, i.e. for $\omega_{n} \neq 0$ or $\mathbf{p} \sim T$, we see that the correction $\sim g^{2} T^{2}$ is a perturbative correction at weak coupling. However, for soft momenta, i.e. for $\omega_{n}=0$ or $\mathbf{p} \sim g T$, the correction is as large as the tree-level term. This is the simplest example of a hard thermal loop (HTL) [20], namely a loop correction which is dominated by hard loop momenta on the order $T$ and is as large as a tree-level term. In this case, the HTL is a simple local mass term. The fact that this term is as large as the tree-level term suggests that we need to reorganize perturbation theory at high temperature.

Screened perturbation theory (SPT) $[21,22,23,24,25]$ is one way of reorganizing the perturbative series, which was inspired by variational perturbation theory [26, 27, 28]. It is defined by writing the Lagrangian as follows

$$
\mathscr{L}_{0}=\frac{1}{2}\left(\partial_{\mu} \phi\right)^{2}+\frac{1}{2} m^{2} \phi^{2}
$$




$$
\mathscr{L}_{\text {int }}=-\frac{1}{2} m^{2} \phi^{2}+\frac{g^{2}}{24} \phi^{4} .
$$

The perturbative expansion is then an expansion around an ideal gas of massive particles. In other words, we incorporate a (thermal) mass to all loop orders via the propagator. In order to avoid overcounting of Feynman diagrams, we need to subtract the quadratic term and consider it as an interaction on the same footing as the quartic interaction. The Feynman rules for SPT are shown in Fig. 3
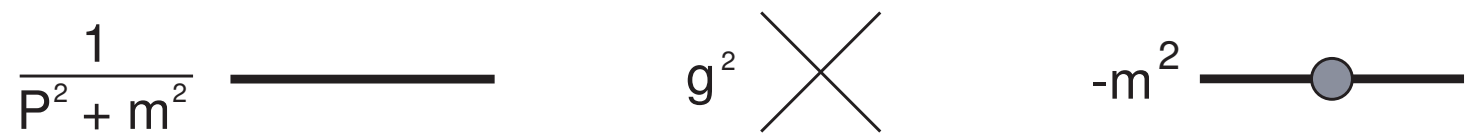

FIGURE 3. Feynman rules in screened perturbation theory.

Using a massive propagator, all infrared divergences are screened and one can, in principle, calculate Feynman diagrams at any order in screened perturbation theory. However, at this stage the mass parameter $m$ in the Lagrangian is arbitrary and in order to complete a calculation in SPT, we need a prescription for it. I will return to this point later in my talk.

\section{Hard-thermal-loop perturbation theory}

Hard-thermal-loop perturbation theory is a generalization of SPT to gauge theories and was developed by Andersen, Braaten, and Strickland over a decade ago [29]. In gauge theories one cannot simply add and subtract a local mass term for the gluons as this would violate gauge invariance.

Looking more carefully at the gluon self-energy function at one-loop order, one realizes that there are contributions (see Fig. 4) which are as large as the tree-level term for soft external momenta. The corresponding loop integral is again dominated by hard momenta and is another example of a hard thermal loop. The contribution to the self-energy

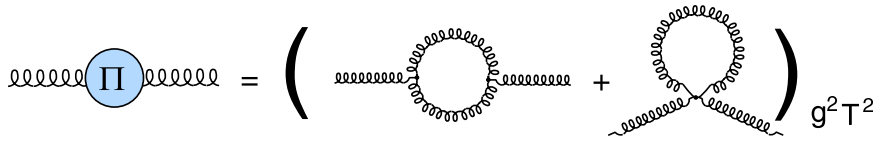

FIGURE 4. Hard thermal loop in nonabelian gauge theory.

function $\Pi$ from the one-loop diagram is then used to construct an effective two-point function, as shown in Fig. 5.

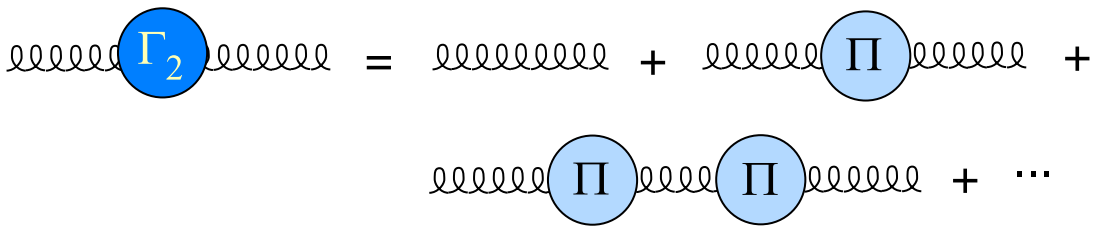

FIGURE 5. Inverse propagator to one-loop order.

It turns out that not only the two-point function receives loop corrections that are as a large as the tree-level contribution, but also higher $n$-point functions do. Thus, we need to use effective vertices together with effective propagators, see Fig. 6. This is essential in order to maintain gauge invariance.

There is a nonlocal effective action that generated all the hard-thermal-loop $n$-point functions [30, 31]. It can be written in a manifestly gauge invariant form and reads

$$
\mathscr{L}_{\mathrm{HTL}}=-\frac{1}{2}(1-\delta) m_{D}^{2} \operatorname{Tr}\left(G_{\mu \alpha}\left\langle\frac{y^{\alpha} y_{\beta}}{(y \cdot D)^{2}}\right\rangle_{\hat{\mathbf{y}}} G^{\mu \beta}\right)+(1-\delta) i m_{q}^{2} \bar{\psi} \gamma^{\mu}\left\langle\frac{y_{\mu}}{y \cdot D}\right\rangle_{\hat{\mathbf{y}}} \psi,
$$

where $G_{\mu \nu}$ is the field strength, $D$ is the covariant derivative, and $m_{D}$ and $m_{q}$ are the Debye screening mass and fermion mass parameters. Moreover, $y=(1, \hat{\mathbf{y}})$ is lightlike four-vector with $\hat{\mathbf{y}}$ being a unit three-vector. The bracket \langle\rangle$_{\hat{\mathbf{y}}}$ indicates 

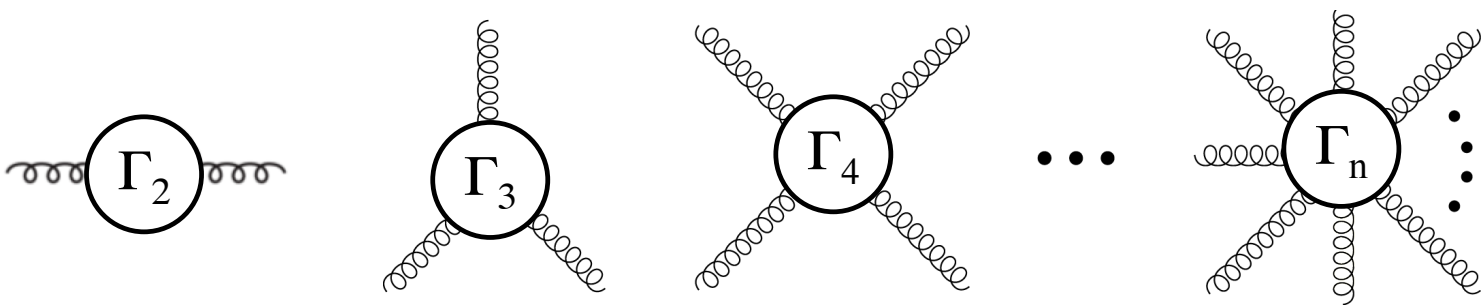

FIGURE 6. Effective $n$-point functions in gauge theories.

an angular average over the directions of $\hat{\mathbf{y}}$. The QCD Lagrangian is then reorganized by writing

$$
\mathscr{L}=\left.\left(\mathscr{L}_{\mathrm{QCD}}+\mathscr{L}_{\mathrm{HTL}}\right)\right|_{g \rightarrow g \sqrt{\delta}}+\Delta \mathscr{L}_{\mathrm{HTL}}
$$

where the QCD Lagrangian in Minkowski space is

$$
\mathscr{L}_{\mathrm{QCD}}=-\frac{1}{2} \operatorname{Tr}\left[G_{\mu \nu} G^{\mu v}\right]+i \bar{\psi} \gamma^{\mu} D_{\mu} \psi+\mathscr{L}_{\mathrm{gh}}+\mathscr{L}_{\mathrm{gf}}+\Delta \mathscr{L}_{\mathrm{QCD}}
$$

The term $\Delta \mathscr{L}_{\mathrm{QCD}}$ contains counterterms necessary to cancel the ultraviolet divergences in perturbative calculations, and $\Delta \mathscr{L}_{\mathrm{HTL}}$ contains the counterterms necessary to cancel additional ultraviolet divergences generated by HTLpt. $\mathscr{L}_{\text {gh }}$ is the ghost term that depends on the gauge-fixing term $\mathscr{L}_{\mathrm{gf}}$, however we emphasize that HTLpt is a gauge invariant framework by construction. It can be used to calculate both static and dynamical quantities. We point out, however, that HTLpt suffers from the same infrared problems related to the magnetic mass as does perturbative QCD, cf. Linde's problem [32]. HTLpt systematically shifts the perturbative expansion from being around an ideal gas of massless particles to being around a gas of massive quasiparticles which are the appropriate physical degrees of freedom at high temperature and/or chemical potential.

Note that the HTLpt Lagrangian (6) reduces to the QCD Lagrangian (7) if we set $\delta=1$. The parameter $\delta$ is an expansion parameter and HTLpt is defined by an expansion in powers of $\delta$ around $\delta=0$. For example at leading order in $\delta, \delta^{0}$, HTLpt describes free massive quasiparticles that include screening effects and Landau damping. At higher orders in $\delta$, we include interactions among these quasiparticles.

In Fig. 7 we show the diagrams contributing to the pressure at order $\delta^{0}, \delta$, and $\delta^{2}$ (left). The different insertions are shown to the right.

A three-loop calculation at finite temperature and with a separate quark chemical potential for each quark yields [18, 19].

$$
\begin{aligned}
\frac{\Omega_{\mathrm{NNLO}}}{\Omega_{0}}= & \frac{7}{4} \frac{d_{F}}{d_{A}} \frac{1}{N_{f}} \sum_{f}\left(1+\frac{120}{7} \hat{\mu}_{f}^{2}+\frac{240}{7} \hat{\mu}_{f}^{4}\right)-\frac{s_{F} \alpha_{s}}{\pi} \frac{1}{N_{f}} \sum_{f}\left[\frac{5}{8}\left(1+12 \hat{\mu}_{f}^{2}\right)\left(5+12 \hat{\mu}_{f}^{2}\right)\right. \\
& \left.-\frac{15}{2}\left(1+12 \hat{\mu}_{f}^{2}\right) \hat{m}_{D}-\frac{15}{2}\left(2 \ln \frac{\hat{\Lambda}}{2}-1-\aleph\left(z_{f}\right)\right) \hat{m}_{D}^{3}+90 \hat{m}_{q}^{2} \hat{m}_{D}\right] \\
& +\frac{s_{2 F}}{N_{f}}\left(\frac{\alpha_{s}}{\pi}\right)^{2} \sum_{f}\left[\frac { 1 5 } { 6 4 } \left\{35-32\left(1-12 \hat{\mu}_{f}^{2}\right) \frac{\zeta^{\prime}(-1)}{\zeta(-1)}+472 \hat{\mu}_{f}^{2}+1328 \hat{\mu}_{f}^{4}\right.\right. \\
& \left.+64\left(-36 i \hat{\mu}_{f} \aleph\left(2, z_{f}\right)+6\left(1+8 \hat{\mu}_{f}^{2}\right) \aleph\left(1, z_{f}\right)+3 i \hat{\mu}_{f}\left(1+4 \hat{\mu}_{f}^{2}\right) \aleph\left(0, z_{f}\right)\right)\right\} \\
& \left.-\frac{45}{2} \hat{m}_{D}\left(1+12 \hat{\mu}_{f}^{2}\right)\right] \\
& +\left(\frac{s_{F} \alpha_{s}}{\pi}\right)^{2} \frac{1}{N_{f}} \sum_{f} \frac{5}{16}\left[96\left(1+12 \hat{\mu}_{f}^{2}\right) \frac{\hat{m}_{q}^{2}}{\hat{m}_{D}}+\frac{4}{3}\left(1+12 \hat{\mu}_{f}^{2}\right)\left(5+12 \hat{\mu}_{f}^{2}\right) \ln \frac{\hat{\Lambda}}{2}\right. \\
& +\frac{1}{3}+4 \gamma_{E}+8\left(7+12 \gamma_{E}\right) \hat{\mu}_{f}^{2}+112 \mu_{f}^{4}-\frac{64}{15} \frac{\zeta^{\prime}(-3)}{\zeta(-3)}-\frac{32}{3}\left(1+12 \hat{\mu}_{f}^{2}\right) \frac{\zeta^{\prime}(-1)}{\zeta(-1)} \\
& \left.-96\left\{8 \aleph\left(3, z_{f}\right)+12 i \hat{\mu}_{f} \aleph\left(2, z_{f}\right)-2\left(1+2 \hat{\mu}_{f}^{2}\right) \aleph\left(1, z_{f}\right)-i \hat{\mu}_{f} \aleph\left(0, z_{f}\right)\right\}\right]
\end{aligned}
$$




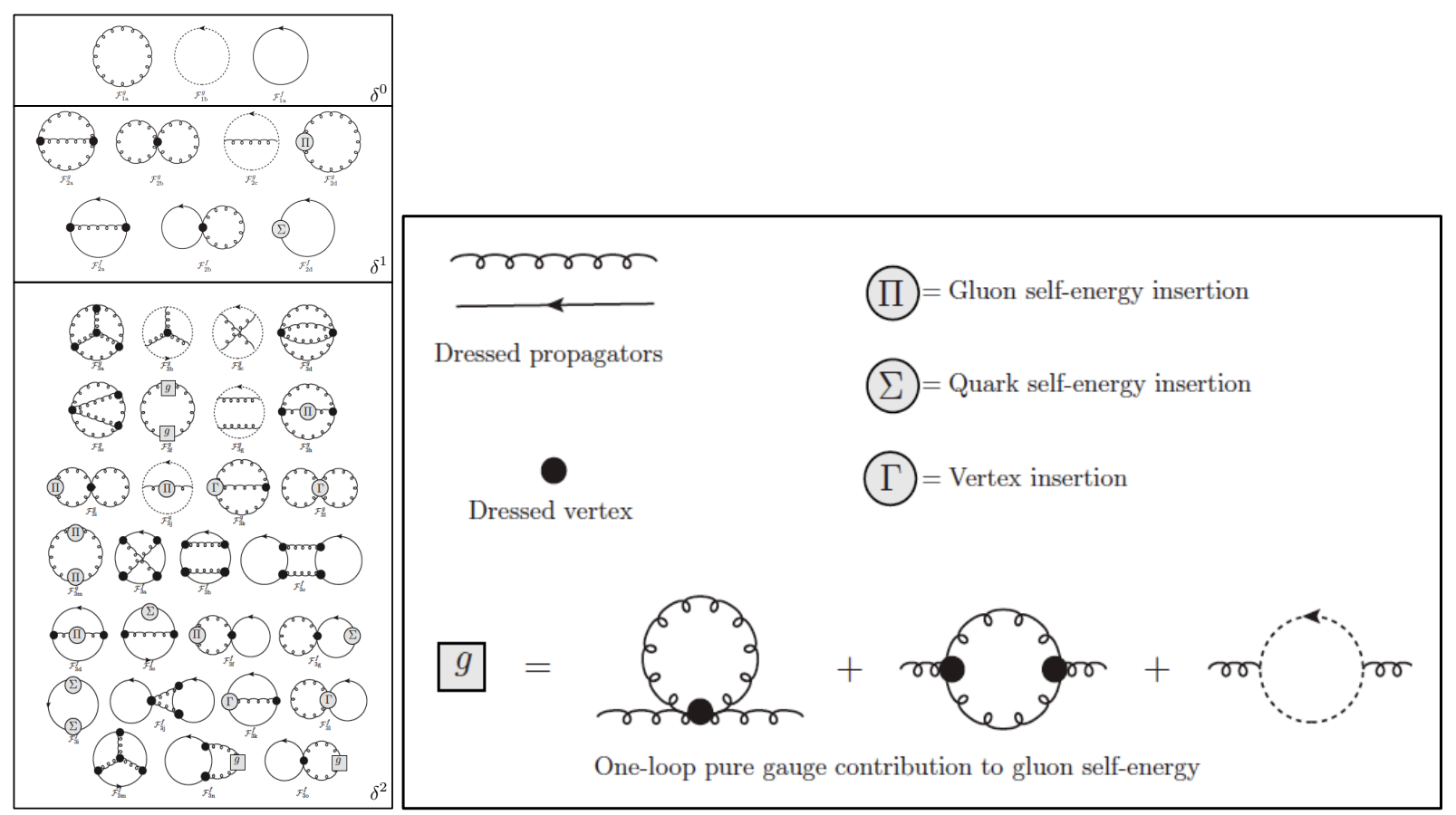

FIGURE 7. Feynman diagrams that contribute to the free energy through next-to-next-to-leading order (left) and various insertions (right).

$$
\begin{aligned}
& +\left(\frac{s_{F} \alpha_{s}}{\pi}\right)^{2} \frac{1}{N_{f}^{2}} \sum_{f, g}\left[\frac{5}{4 \hat{m}_{D}}\left(1+12 \hat{\mu}_{f}^{2}\right)\left(1+12 \hat{\mu}_{g}^{2}\right)+90\left\{2\left(1+\gamma_{E}\right) \hat{\mu}_{f}^{2} \hat{\mu}_{g}^{2}\right.\right. \\
& -\left\{\aleph\left(3, z_{f}+z_{g}\right)+\aleph\left(3, z_{f}+z_{g}^{*}\right)+4 i \hat{\mu}_{f}\left[\aleph\left(2, z_{f}+z_{g}\right)+\aleph\left(2, z_{f}+z_{g}^{*}\right)\right]-4 \hat{\mu}_{g}^{2} \aleph\left(1, z_{f}\right)\right. \\
& \left.\left.-\left(\hat{\mu}_{f}+\hat{\mu}_{g}\right)^{2} \aleph\left(1, z_{f}+z_{g}\right)-\left(\hat{\mu}_{f}-\hat{\mu}_{g}\right)^{2} \aleph\left(1, z_{f}+z_{g}^{*}\right)-4 i \hat{\mu}_{f} \hat{\mu}_{g}^{2} \aleph\left(0, z_{f}\right)\right\}\right\} \\
& \left.-\frac{15}{2}\left(1+12 \hat{\mu}_{f}^{2}\right)\left(2 \ln \frac{\hat{\Lambda}}{2}-1-\aleph\left(z_{g}\right)\right) \hat{m}_{D}\right] \\
& +\left(\frac{c_{A} \alpha_{s}}{3 \pi}\right)\left(\frac{s_{F} \alpha_{s}}{\pi N_{f}}\right) \sum_{f}\left[\frac{15}{2 \hat{m}_{D}}\left(1+12 \hat{\mu}_{f}^{2}\right)-\frac{235}{16}\left\{\left(1+\frac{792}{47} \hat{\mu}_{f}^{2}+\frac{1584}{47} \hat{\mu}_{f}^{4}\right) \ln \frac{\hat{\Lambda}}{2}\right.\right. \\
& -\frac{144}{47}\left(1+12 \hat{\mu}_{f}^{2}\right) \ln \hat{m}_{D}+\frac{319}{940}\left(1+\frac{2040}{319} \hat{\mu}_{f}^{2}+\frac{38640}{319} \hat{\mu}_{f}^{4}\right)-\frac{24 \gamma_{E}}{47}\left(1+12 \hat{\mu}_{f}^{2}\right) \\
& -\frac{44}{47}\left(1+\frac{156}{11} \hat{\mu}_{f}^{2}\right) \frac{\zeta^{\prime}(-1)}{\zeta(-1)}-\frac{268}{235} \frac{\zeta^{\prime}(-3)}{\zeta(-3)}-\frac{72}{47}\left[4 i \hat{\mu}_{f} \aleph\left(0, z_{f}\right)+\left(5-92 \hat{\mu}_{f}^{2}\right) \aleph\left(1, z_{f}\right)\right. \\
& \left.\left.+144 i \hat{\mu}_{f} \aleph\left(2, z_{f}\right)+52 \aleph\left(3, z_{f}\right)\right]\right\}+90 \frac{\hat{m}_{q}^{2}}{\hat{m}_{D}}+\frac{315}{4}\left\{\left(1+\frac{132}{7} \hat{\mu}_{f}^{2}\right) \ln \frac{\hat{\Lambda}}{2}\right. \\
& \left.\left.+\frac{11}{7}\left(1+12 \hat{\mu}_{f}^{2}\right) \gamma_{E}+\frac{9}{14}\left(1+\frac{132}{9} \hat{\mu}_{f}^{2}\right)+\frac{2}{7} \aleph\left(z_{f}\right)\right\} \hat{m}_{D}\right]+\frac{\Omega_{\mathrm{NNLO}}^{\mathrm{YM}}}{\Omega_{0}},
\end{aligned}
$$

where the sums over $f$ and $g$ include all quark flavors, $z_{f}=1 / 2-i \hat{\mu}_{f}, \Omega_{\mathrm{NNLO}}^{\mathrm{YM}}$ is the pure-glue contribution [33], $\Omega_{0}=-d_{A} \pi^{2} T^{4} / 45, \hat{m}_{D}=m_{D} /(2 \pi T)$ etc, and $\alpha_{s}=g^{2} /(4 \pi)$. Moreover $\aleph(z)=\Psi(z)+\Psi\left(z^{*}\right)$, where $\Psi(z)$ is the digamma function and $\boldsymbol{\aleph}(n, z)=\zeta^{\prime}(-n, z)+(-1)^{n+1} \zeta^{\prime}\left(-n, z^{*}\right)$ where $\zeta^{\prime}(x, y)=\partial_{x} \zeta(x, y) . c_{A}=N_{c}, d_{A}=N_{c}^{2}-1$, $s_{F}=\frac{1}{2} N_{f}, s_{2 F}=C_{F} s_{F}$ with $C_{F}=\frac{N_{c}^{2}-1}{2 N_{c}}$. Note that there are two renormalization scales $\Lambda_{g}$ and $\Lambda_{q}$, we use former in 
the purely gluonic graphs and the latter in all other graphs. In this way the susceptibilities vanish in the limit $N_{f} \rightarrow 0$. In addition, the thermodynamic potential produces the correct $\mathscr{O}\left(g^{5}\right)$ weak-coupling result if expanded in a strict power series in $g$.

\section{Dimensional reduction}

In scalar field theory, we have seen that the resummed effective propagator is of the form $1 /\left(P^{2}+m^{2}\right)$, where $m$ is on the order $g T$. There are two momentum scales in scalar theory (and in QED), namely the hard scale $T$ and the soft scale $g T$ ( $e T$, where $e$ is the electric charge). At weak coupling, the term $m^{2}$ is a perturbative correction to the propagator for the nonzero Matsubara modes, while it is essential to include for the static mode $P_{0}=0$. At weak coupling, one therefore has to well separated mass scales and one can integrate out the nonzero Matsubara frequencies to obtain an effectice three-dimensional field theory for the zeroth Matsubara mode [34, 35]. The idea is shown in Fig. 8, where the imaginary time dimension is $1 / T$ and so the system becomes effectively three-dimensional at high temperature.

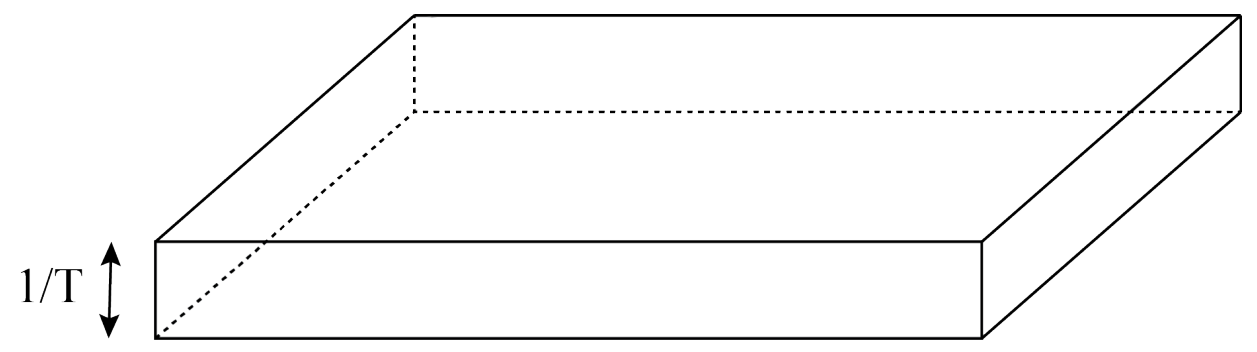

FIGURE 8. Dimensional reduction in hot field theories.

The scale $T$ can be integrated out perturbatively and in the case of scalar $\phi^{4}$-theory, the effective three-dimensional Lagrangian becomes

$$
\mathscr{L}_{\text {eff }}=\frac{1}{2}(\nabla \phi)^{2}+\frac{1}{2} m_{3}^{2} \phi^{2}+\frac{\lambda}{24} \phi^{4}+\delta \mathscr{L},
$$

where $\delta \mathscr{L}$ contains higher-order operators. The parameters of the effective Lagrangian are functions of $T, g^{2}$, and a renormalization scale $\Lambda$. For example $m_{3}^{2}=\frac{g^{2}}{24} T^{2}$ and $\lambda=g^{2} T$ to leading order in $g^{2}$. In QED, dimensional reduction can be carried out the same way and one obtains an effective three-dimensional theory for a massive scalar field $A_{0}$, which can be identified with the zeroth component of the original gauge field, and a gauge field $A_{i}$, which can identified with the spatial components of the original gauge field ${ }^{2}$. In QCD, it is somewhat more complicated as we have three momentum scales, namely the hard scale $T$, soft scale $g T$, and the supersoft scale $g^{2} T$. At weak coupling, we then have three well separated momentum scales and we can successive integrate out the scales $T$ and $g T$. Integrating out the scale $T$, we obtain an effective three-dimensional theory, Electrostatic QCD (EQCD), whose Lagrangian is

$$
\mathscr{L}_{\mathrm{EQCD}}=\frac{1}{2} \operatorname{Tr}\left[G_{i j}\right]+\operatorname{Tr}\left[\left(D_{i} A_{0}\right)^{2}\right]+M_{E}^{2} \operatorname{Tr}\left[A_{0}^{2}\right]+i \xi \operatorname{Tr}\left[A_{0}^{3}\right]+\lambda_{E} \operatorname{Tr}\left[A_{0}^{4}\right]+\delta \mathscr{L},
$$

where $G_{i j}$ is the nonabelian field strength tensor and $A_{0}$ is a scalar field in the adjoint representation of the gauge group $S U$ (3). Moreover, $m_{E}$ is the color electric screening mass, $\lambda_{E}$ is the scalar self-coupling, and $\xi \propto \sum_{f} \mu_{f}$ [36]. The term $\delta \mathscr{L}$ contains all higher-order operators that start to contribute to the pressure beyond order $g^{6}$. We note in passing that EQCD breaks the $S U\left(N_{c}\right)$ center symmetry of four-dimensinonal QCD, which can be remedied by formulating the theory in terms of Wilson loop type variables instead of the $A_{0}$ field $[37,38]$. However, for high temperatures, this is of little consequence as the probability of thermal fluctuations crossing the barriers that separate the trivial minimum we are expanding about, and other minima is exponentially small.

Dimensional reduction has been carried out both at zero density $[39,40]$ as well as at finite density [11] to order $g^{6} \ln (g)$. The color electric screening mass is on the order $g T$, and as a result, we integrate it out perturbatively to obtain

\footnotetext{
${ }^{2}$ The fermions are massive since $\omega_{n}=(2 n+1) \pi T$ so they are integrated out as well.
} 
a second effective field theory (Magnetostatic QCD). This theory is plagued with infrared divergences in perturbation theory and must be treated nonperturbatively using lattice simulations. We can now write the pressure in QCD as a sum of contributions from the different momentum scales

$$
\mathscr{P}=\mathscr{P}_{\text {hard }}+\mathscr{P}_{\text {soft }}+\mathscr{P}_{\text {supersoft }} \text {. }
$$

The contribution from the hard scale comes from dimensional reduction (which can be identified with the unit operator in $\mathscr{L}_{\mathrm{EQCD}}$ ). The contribution from the soft scale comes from calculations in using EQCD, while the contribution from the supersoft scale comes from calculations in MQCD. However, MQCD contributes first at order $g^{6}$, and as a result, we can ignore these contributions if we restrict ourselves to order $g^{5}$.

\section{NUMERICAL RESULTS}

We next present our numerical results. Physical quantities such as the pressure and susceptibilities depend on the mass parameters $m_{D}$ and $m_{f}$ as well as the running coupling $\alpha_{s}$ and the renormalization scales $\Lambda_{g}$ and $\Lambda_{q}$ We fixed the scale $\overline{\mathrm{MS}}$ by requiring that $\alpha_{s}(1.5 \mathrm{GeV})=0.326$, which is obtained from lattice measurements [41]. Using one-loop running this gives $\overline{\mathrm{MS}}=176 \mathrm{MeV}$, while two-loop running gives $\overline{\mathrm{MS}}=283 \mathrm{MeV}$. We used one-loop running for HTLpt and two-loop running for DR. We take the central values $\Lambda_{g}=2 \pi T$ and $\Lambda_{q}=2 \pi \sqrt{T^{2}+\mu^{2} / \pi^{2}}$ in our HTL calculations. In our DR calculations, we used a central value of $\Lambda=1.445 \times 2 \pi T$ for $\mu_{q}=0$, which is based on the principle of fastest apparent convergence. This can be generalized to nonzero density, see Ref. [17] for details. In all plots, the thick lines indicate the results obtained using these central values and the bands are obtained by varying the scales by a factor of two. For the numerical results presented in this talk, we use $c_{A}=N_{c}$ and $N_{f}=3$.

As mentioned before, one needs to give a prescription for the mass parameters $m_{D}$ and $m_{f}$. One can think of a variational prescription such as

$$
\frac{\partial \mathscr{F}}{\partial m_{D}^{2}}=0
$$

where $\mathscr{F}$ is the free energy. At one-loop order in HTLpt, the only solution is the trivial solution. At higher orders, this equation has complex solution for some values of the coupling. We therefore decided to use value $m_{f}=0$ for the fermion mass parameter and the Debye mass $m_{D}$ given by the two-loop expression for the mass parameter in EQCD [11],

$$
\begin{aligned}
\hat{m}_{D}^{2}= & \frac{\alpha_{s}}{3 \pi}\left\{c_{A}+\frac{c_{A}^{2} \alpha_{s}}{12 \pi}\left(5+22 \gamma_{E}+22 \ln \frac{\hat{\Lambda}_{g}}{2}\right)+\frac{1}{N_{f}} \sum_{f}\left[s_{F}\left(1+12 \hat{\mu}_{f}^{2}\right)+\frac{c_{A} s_{F} \alpha_{s}}{12 \pi}\left(\left(9+132 \hat{\mu}_{f}^{2}\right)\right.\right.\right. \\
& \left.+22\left(1+12 \hat{\mu}_{f}^{2}\right) \gamma_{E}+2\left(7+132 \hat{\mu}_{f}^{2}\right) \ln \frac{\hat{\Lambda}}{2}+4 \aleph\left(z_{f}\right)\right) \\
& \left.\left.+\frac{s_{F}^{2} \alpha_{s}}{3 \pi}\left(1+12 \hat{\mu}_{f}^{2}\right)\left(1-2 \ln \frac{\hat{\Lambda}}{2}+\aleph\left(z_{f}\right)\right)-\frac{3}{2} \frac{s_{2 F} \alpha_{s}}{\pi}\left(1+12 \hat{\mu}_{f}^{2}\right)\right]\right\} .
\end{aligned}
$$

When we evaluate various physical quantities either in HTLpt or DR, it is essential that we do not expand the mass parameter $m_{D}$ in powers of $g$, but keep the full $g$-dependence to resum the perturbative series.

\section{Pressure}

In Fig. 9, we show the normalized pressure as a function of $T$ for $\mu_{B}=0$ (left) and $\mu_{B}=400 \mathrm{MeV}$ (right). The solid lines are obtained by using the central value $\Lambda_{g}=2 \pi T$ and $\Lambda_{q}=2 \pi \sqrt{T^{2}+\mu^{2} / \pi^{2}}$ for the renormalization scales. The blue bands are obtained by varying this scale around the central value by a factor of two. The lattice data are from the Budapest-Wuppertal collaboration [42,43]. The agreement with the central line and the lattice data is remarkable all the way down to approximately $T=200 \mathrm{MeV}$. Since HTLpt is a perturbative approach that does not incorporate the $S U\left(N_{c}\right)$ center symmetry, there is no reason to expect agreement with the data at temperatures close to $T_{c}$ and the agreement seen may be fortuitous. 

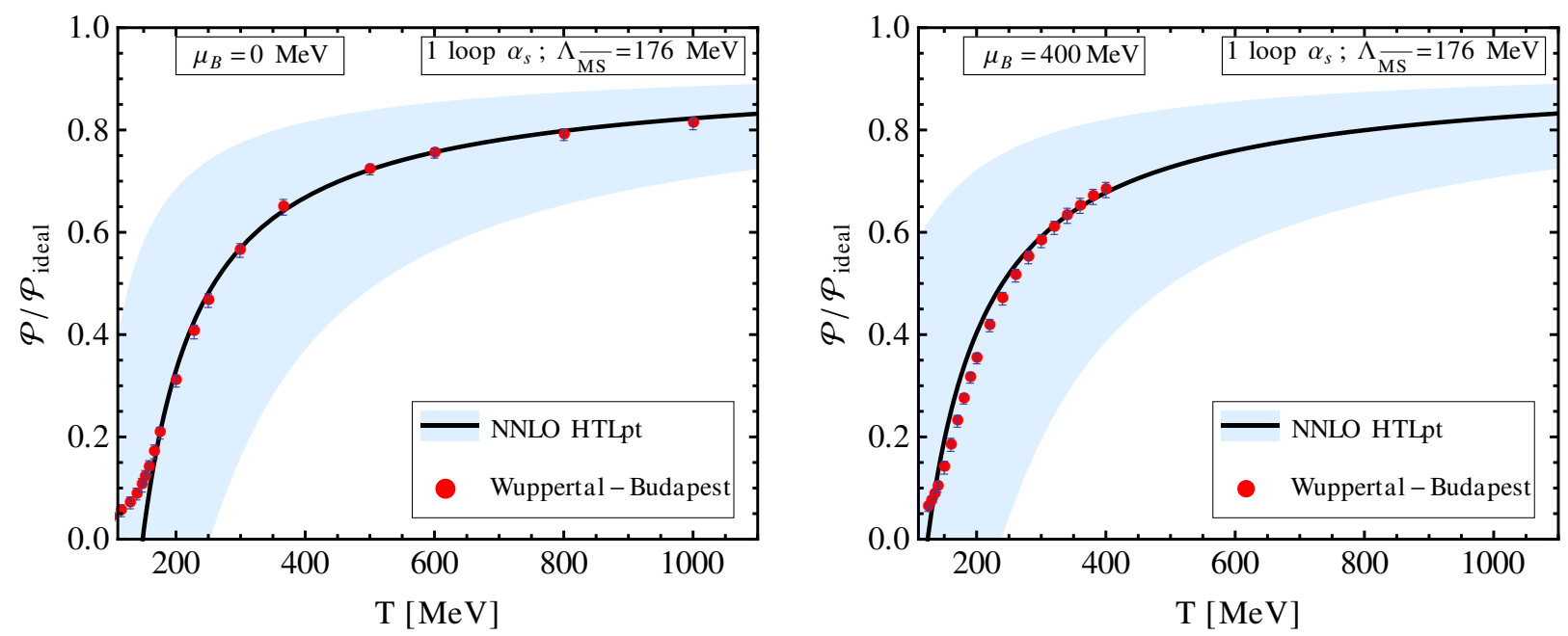

FIGURE 9. The pressure normalized to the pressure of an ideal gas of massless particles as a function of temperature $T$. Lattice data are from [42] and [43], respectively.

\section{Susceptibilities}

We next turn to the quark and baryon susceptibilities. The pressure is a function of the temperature $T$ and the quark chemical potentials $\mu_{q}$. Furthermore, we can expand the pressure as a Taylor series in powers of $\mu_{q} / T$ around zero chemical potential:

$$
\frac{\mathscr{P}}{T^{4}}=\frac{\mathscr{P}_{0}}{T^{4}}+\sum_{i j k} \frac{1}{i ! j ! k !} \chi_{i j k}\left(\frac{\mu_{u}}{T}\right)^{i}\left(\frac{\mu_{d}}{T}\right)^{j}\left(\frac{\mu_{s}}{T}\right)^{k}
$$

where the coefficients are given by

$$
\chi_{i j k \ldots}=\left.\frac{\partial^{i+j+k+\ldots \mathscr{P}}(T, \mu)}{\partial \mu_{u}^{i} \partial \mu_{d}^{j} \partial \mu_{s}^{k}}\right|_{\mu_{q}=0} .
$$

Below, we will use the shorthand notation for the susceptibilities by specifying derivatives by a string of quark flavors in superscript form, e.g. $\chi_{2}^{u u}=\chi_{200}, \chi_{2}^{d s}=\chi_{011}, \chi_{2}^{u u d d}=\chi_{220}$ etc. We will also interested in the baryon-number susceptibilities, which are defined by

$$
\chi_{n}^{B}=\left.\frac{\partial^{n} \mathscr{P}}{\partial \mu_{B}^{n}}\right|_{\mu_{B}=0}
$$

Using the relation $\mu_{B}=\mu_{u}+\mu_{d}+\mu_{s}$ and the chain rule we can find relations between the quark and baryon susceptibilities. For example, one finds

$$
\chi_{2}^{B}=\frac{1}{9}\left[\chi_{2}^{u u}+\chi_{2}^{d d}+\chi_{2}^{s s}+2 \chi_{2}^{u d}+2 \chi_{2}^{u s}+2 \chi_{2}^{d s}\right]
$$

In Fig. 10, we compare the scaled second (left) and fourth-order (right) baryon number susceptibility from HTLpt and DR compared with various lattice data. Both resummation schemes are in good agreement with the data. We also notice that the band using DR is significantly smaller than that obtained in HTLpt.

In Fig. 11, we show the NNLO HTLpt fourth order diagonal single quark number susceptibility (left) and the only non-vanishing fourth order off-diagonal quark number susceptibility (right) with lattice data. Again the agreement between the HTLpt prediction and the lattice data is good. 

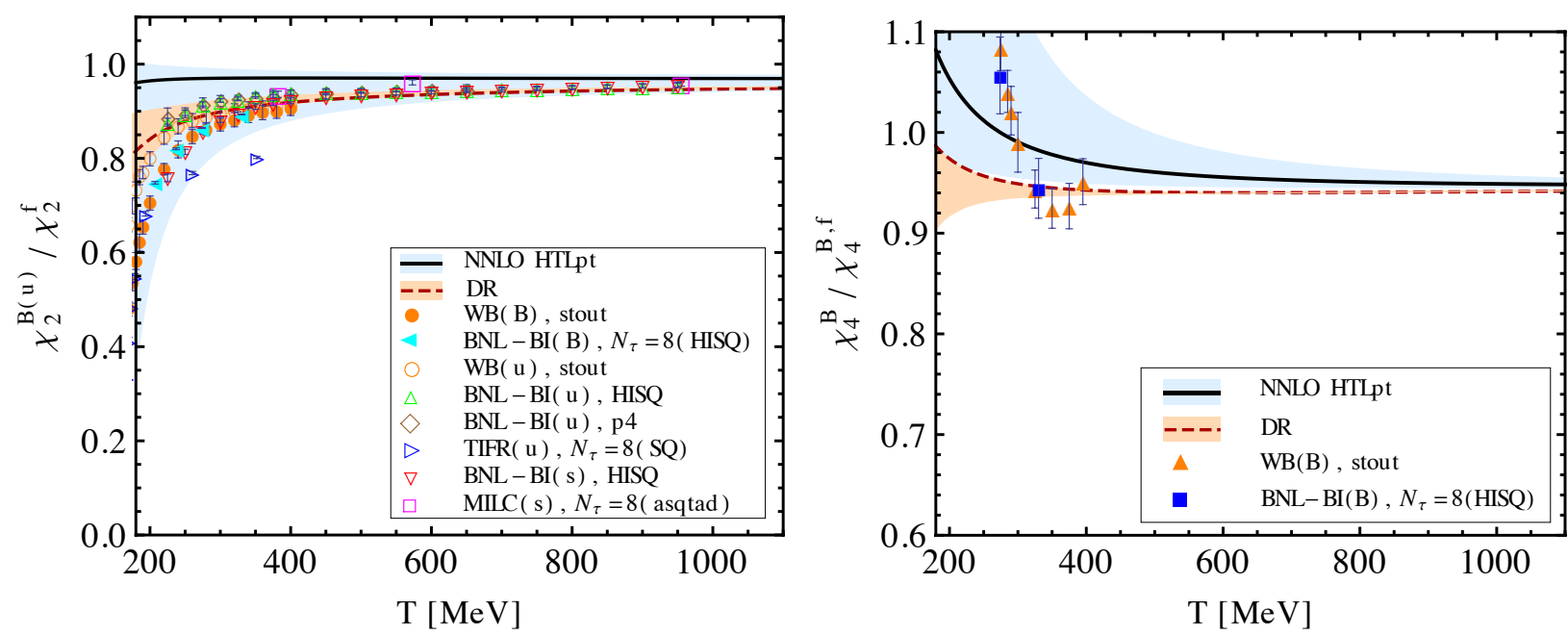

FIGURE 10. The scaled second (left) and fourth-order (right) baryon number susceptibility from HTLpt and DR compared with various lattice data. The lattice data labeled WB, BNL-BI(B), BNL-BI(u,s), MILC, and TIFR come from Refs. [44, 45, 46, 47, 48].
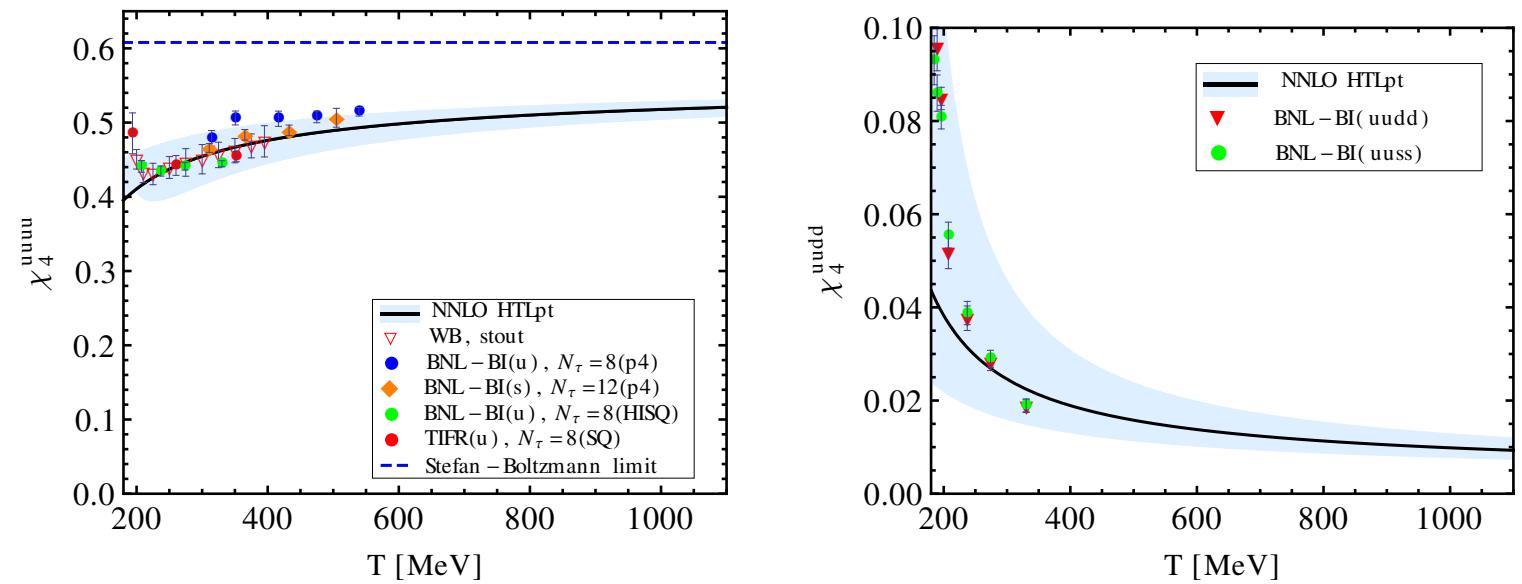

FIGURE 11. Comparison of the NNLO HTLpt fourth order diagonal single quark number susceptibility (left) and the only nonvanishing fourth order off-diagonal quark number susceptibility (right) with lattice data. In the left figure the dashed blue line indicates the Stefan-Boltzmann limit for this quantity. The data labeled BNL-BI(uudd), BNL-BI(u,s), BNL-BI(uuss), and TIFR come from Refs. [45, 46, 47, 48].

\section{Interaction measure and speed of sound}

The interaction measure is given by

$$
\mathscr{E}-3 \mathscr{P},
$$

where $\mathscr{E}$ is the energy density. Since this quantity is the trace of the energy-momentum tensor and vanishes for an ideal gas of massless particles, it is also referred to as the trace anomaly. In Fig. 12, we show the interaction measure normalized by $T^{4}$ as a function of temperature $T$ for $\mu_{B}=0$ (upper left panel) and $\mu_{B}=400 \mathrm{MeV}$ (upper right panel). The lattice data are from the Wuppertal-Budapest collaboration [42, 43]. We see that the agreeement between the prediction from HTLpt and lattice data is very good all the way down to $T \approx 250 \mathrm{MeV}$, which is very near where the peak is located. For lower values of the temperature, HTLpt fails completely, but this is not very surprising. There is no reason to expect that resummed perturbation theory works close to the QCD transition temperature. 

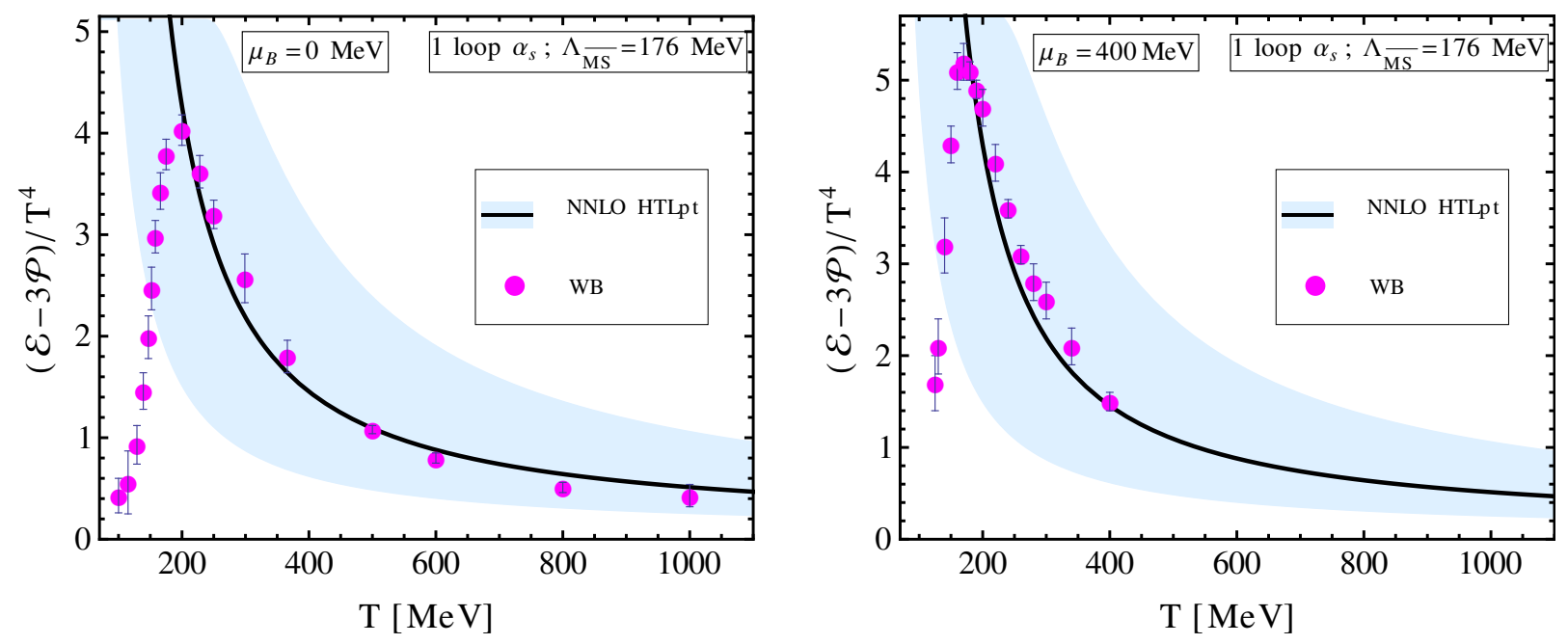

FIGURE 12. Interaction measure $\mathscr{E}-3 \mathscr{P}$ divided by $T^{4}$ as a function of temperature $T$ for $\mu_{B}=0$ (left) and $\mu_{B}=400 \mathrm{MeV}$ (right). The $\mu_{B}=0$ lattice data are from [42] and the $\mu_{B}=400 \mathrm{MeV}$ lattice data are from [43].
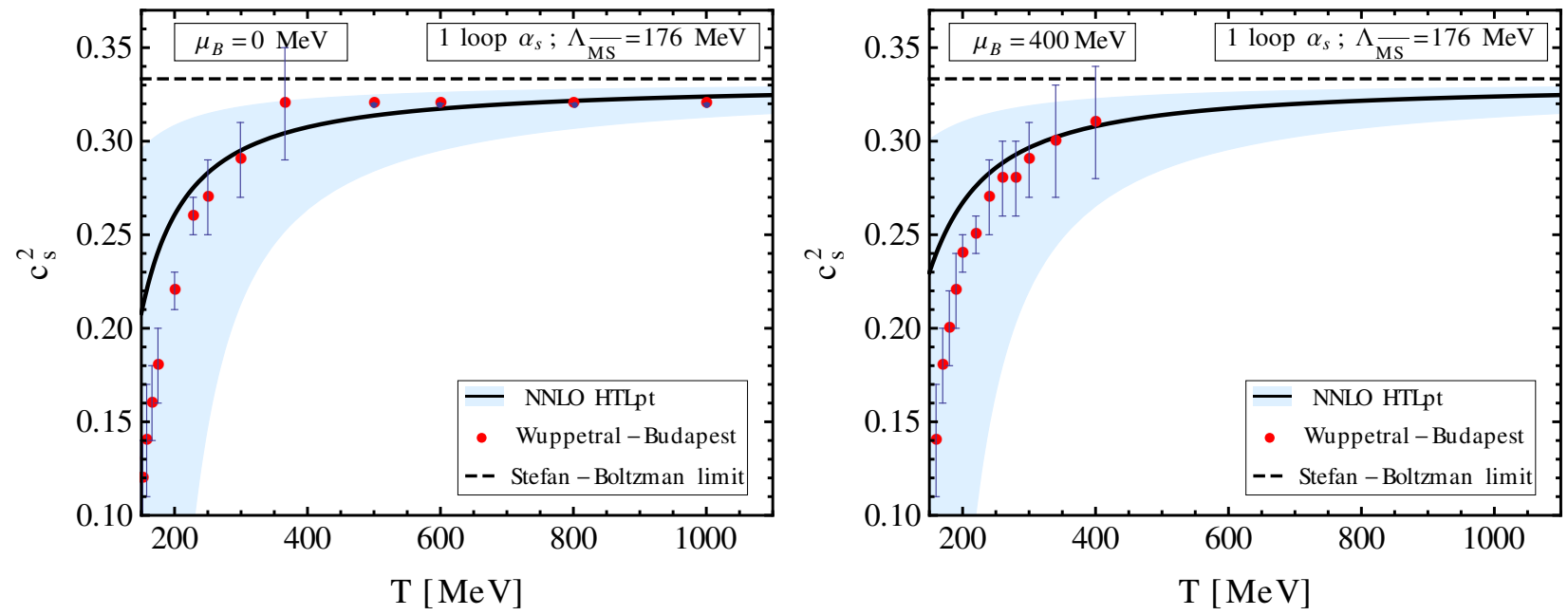

FIGURE 13. Comparison of the $N_{f}=2+1, \mu_{B}=0$ (left) and $\mu_{B}=400 \mathrm{MeV}$ (right) NNLO HTLpt speed of sound squared with lattice data. The $\mu_{B}=0$ lattice data are from [42] and the $\mu_{B}=400 \mathrm{MeV}$ lattice data are from [43]. The dashed lines indicate the Stefan-Boltzmann limit.

Another quantity which is phenomenologically interesting is the speed of sound. The speed of sound squared is defined as

$$
c_{s}^{2}=\frac{\partial \mathscr{P}}{\partial \mathscr{E}} .
$$

In Fig. 13 we plot the NNLO HTLpt speed of sound squared for $\mu_{B}=0$ (left) and $\mu_{B}=400 \mathrm{MeV}$ (right) together with lattice data from Refs. [42] and [43]. As we can see from this figure, there is quite good agreement between the NNLO HTLpt speed of sound and the lattice data when the central value of the scale is used. 


\section{SUMMARY AND OUTLOOK}

In this talk, I have presented results for QCD thermodynamic functions using hard-thermal-loop perturbation theory and dimensional reduction. We note that our results are completely analytic and that, after we have chosen the renormalization scales $\Lambda_{g}$ and $\Lambda_{q}$, there are no fit parameters. Comparing our results with available lattice data, they suggest that for temperatures above $250-500 \mathrm{MeV}$ (depending on the quantity we are looking at), the pressure, quark number susceptibilities, and other quantities can be accurately described via resummed perturbation theory.

Hard-thermal-loop perturbation theory represents a gauge-invariant reorganization of the perturbative series. It is formulated in Minkowski space and so can be used to calculate static and dynamical quantities alike. The good agreement between HTLpt and lattice offers some hope that applications to real-time quantities will be useful.

There are several directions for future work. One is to include finite quark masses, another is to resum logarithms to reduce the scale variation of our results. Extending our results to larger values of $\mu_{q}$ is also of interest as there currently no other first principle method is available.

\section{ACKNOWLEDGMENTS}

The speaker would like to thank the organizers for an interesting and stimulating meeting. He also thanks his coauthors for an enjoyable collaboration. The authors thank S. Borsányi, S. Datta, F. Karsch, S. Gupta, S. Mogliacci, P. Petreczky, and A. Vuorinen for useful discussions.

\section{REFERENCES}

1. E. V. Shuryak, Sov. Phys. JETP 47, 212-219 (1978).

2. S. A. Chin, Phys. Lett. B 78, 552-555 (1978).

3. J. I. Kapusta, Nucl. Phys. B 148, 461-498 (1979).

4. T. Toimela, Phys. Lett. B 124, 407-409 (1983).

5. P. Arnold, C. X. Zhai, Phys. Rev. D 50, 7603-7623 (1994).

6. P. Arnold, C. X. Zhai, Phys. Rev. D 51, 1906-1918 (1995).

7. C. X. Zhai and B. M. Kastening, Phys. Rev. D 52, 7232-7246 (1995).

8. E. Braaten, and A. Nieto, Phys. Rev. D 53, 3421-3437 (1996).

9. K. Kajantie, M. Laine, K. Rummukainen and Y. Schröder, Phys. Rev. D 67, 105008 (2003).

10. A. Vuorinen, Phys. Rev. D 67, 074032 (2003).

11. A. Vuorinen, Phys. Rev. D 68, 054017 (2003).

12. A. Ipp, K. Kajantie, A. Rebhan, and A. Vuorinen, Phys. Rev. D 74, 045016 (2006).

13. A. D. Linde, Phys. Lett. B 96, 289-292 (1980).

14. J. O. Andersen and M. Strickland, Ann. Phys. 317, 281-353 (2005).

15. U. Kraemmer and A. Rebhan, Rept. Prog. Phys. 67, 351-431 (2004).

16. J. O. Andersen, L. E. Leganger, M. Strickland, and N. Su, JHEP 08, 053 (2011).

17. S. Mogliacci, J. O. Andersen, M. Strickland, N. Su, and A. Vuorinen, JHEP 12, 055 (2013).

18. N. Haque, J. O. Andersen, M. G. Mustafa, M. Strickland, and N. Su, Phys. Rev. D 89, 061701 (2014).

19. N. Haque, A. Bandyopadhyay, J. O. Andersen, M. G. Mustafa, M. Strickland, and N. Su, JHEP 05, 027 (2014).

20. E. Braaten, and R. D. Pisarski, Nucl. Phys. B, 337, 569-634 (1990).

21. F. Karsch, A. Patkos and P. Petreczky, Phys. Lett. B 401, 69-73, (1997).

22. S. Chiku and T. Hatsuda, Phys. Rev. D 58, 076001 (1998).

23. J. O. Andersen, E. Braaten and M. Strickland, Phys. Rev. D 63, 105008 (2001).

24. J. O. Andersen and L. Kyllingstad, Phys. Rev. D 78, 076008 (2008).

25. J. O. Andersen and M. Strickland, Phys. Rev. D 64, 105012 (2001).

26. V. I. Yukalov, Teor. Mat. Fiz. 26, 403-413, (1976).

27. P. M. Stevenson, Phys. Rev. D 23, 2916-2944 (1981).

28. A. Duncan and M. Moshe, Phys. Lett. B 215, 352-358 (1988).

29. J. O. Andersen, E. Braaten, and M. Strickland, Phys. Rev. Lett. 83, 2139-2142 (1999).

30. J. C. Taylor, and S. M. H. Wong, Nucl. Phys. B 346, 115-128 (1990).

31. E. Braaten, and R. D. Pisarski, Phys. Rev. D 45, 1827-1830 (1992).

32. N. Su and K. Tywoniuk, arXiv:1409.3203 [hep-ph].

33. J. O. Andersen, M. Strickland, and N. Su, JHEP 1008, 113 (2010)

34. E. Braaten, and A. Nieto, Phys. Rev. D, 51, 6990-7006 (1995).

35. K. Kajantie, M. Laine, K. Rummukainen, and M. E. Shaposhnikov, Nucl. Phys. B, 458, 90-136 (1996).

36. A. Hart, M. Laine, and O. Philipsen, Nucl. Phys. B 586, 443-474 (2000) 
37. A. Vuorinen and L. G. Yaffe, Phys. Rev. D 74, 025011 (2006).

38. P. de Forcrand, A. Kurkela and A. Vuorinen, Phys. Rev. D 77, 125014 (2008).

39. K. Kajantie, M. Laine, K. Rummukainen, and Y. Schröder, Phys. Rev. D, 67, 105008 (2003).

40. K. Kajantie, M. Laine, K. Rummukainen, and Y. Schröder, JHEP 04, 036 (2003).

41. A. Bazavov, N. Brambilla, X. Garcia i Tormo, P. Petreczky, J. Soto, and A. Vairo, Phys. Rev. D 86, 114031 (2012).

42. S. Borsányi et al., JHEP 11, 077 (2010).

43. S. Borsányi et al., JHEP 08, 53 (2012).

44. S. Borsányi, Z. Fodor, S. D. Katz, S. Krieg, C. Ratti, and K. Szabo, JHEP 01, 138 (2012).

45. A. Bazavov et al., Phys. Rev. Lett. 111, 082301 (2013).

46. Phys.Rev. D 88, 094021 (2013).

47. C. Bernard et al., Phys. Rev. D 71, 034504 (2005).

48. S. Datta, R.V. Gavai and S. Gupta, PoS LATTICE2013, 202 (2013). 